Military Technical College Kobry El-Kobbah, Cairo, Egypt.

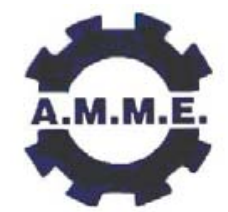

$13^{\text {th }}$ International Conference on Applied Mechanics and Mechanical Engineering.

\title{
CARBON-POLYMER NANOCOMPOSITE ON BASE OF NANOFIBER TEXTILE PRODUCED BY NANOSPIDER METHOD
}

\author{
ROŻEK $^{\star}$ Z., KACZOROWSKI ${ }^{* *}$ W., LUKÁŠ ${ }^{* * *}$ D., LOUDA ${ }^{* * * *}$ P. and MITURA ${ }^{* * * * *}$ S.
}

\begin{abstract}
Due to specific properties of carbon coatings, they have been used in a lot of applications for many years. Plotted on the substrate they mainly fulfill protective function. Currently a lot of attention is dedicated to carbon coatings produced on polymer substrate. In this case plasma enhanced CVD methods play a very important role. Using these methods makes carbon coatings synthesis possible in lower temperature.

Carbon coatings have been plotted on to polymer substrate [poly (vinylalcohol) (PVA), polyurethane (PUR)], by Microwave Radio Frequency Plasma Assisted Chemical Vapour Deposition (MW/RF PA CVD) method. Plasma has been aroused in pure a methane atmosphere. Nanofiber textile has been used as the substrate produced by modern Nanospider method. The Nanospider is the modification of the electrospinning. Nanocomposite obtained in this way was characterized by the contact angle studies. The nanocomposite structure was analyzed by scanning electron microscope (SEM). The results of investigations are presented.
\end{abstract}

\section{KEY WORDS}

Carbon coatings, Nanofibers, Nanocomposites

PhD student, Dpt. of Mater. Sc. and Eng., Technical University, Liberec,

Czech Republic.

** PhD, Dpt. of Mater. Sc. and Eng., Technical University, Lodz, Poland.

*** Professor, Dpt. of Nonwovens., Technical University, Liberec, Czech Republic.

**** Professor, Dpt. of Mater. Sc. and Eng., Technical University, Liberec, Czech Republic.

***** Professor, Dpt. of Mater. Sc. and Eng., Technical University, Lodz, Poland. 


\section{INTRODUCTION}

Carbon coatings deposited by Microwave Radio Frequency Plasma Assisted Chemical Vapor Deposition (MW/RF PACVD) method have been used in various areas for many years. This material is characterized by many unique properties such as high hardness, low coefficient of friction, chemical inertness. All these properties, together with biocompatibility, match well with the criteria of a good biomaterial for applications in orthopedic and dentistry [1,2] or cardiovascular [3]. It has been proved that including diamond phase in carbon coatings guarantees high wear [4] and corrosion [5] resistance of covered implant.

MW/RF PACVD is a suitable method for carbon film deposition on polymers owing to its processing performed at low temperature, avoiding any degradation of polymer substrates. Polymeric materials covered by carbon coatings are also widely used in biomedical fields [6,7]. High biological activity of carbon has been established [8]; this fact has persuaded the authors of this paper to utilize deposition of carbon coatings on nanofiber textile. The results of our first investigations are very promising. Moreover, it has to be underlined that it is our belief that we are first to have deposited carbon coatings on nanofiber textile.

Currently Diamond Powder Particles (DPP) are considered as the new, excellent antioxidant and anti-inflammatory factor in living organism. DPP are characterized by extended surface, and high activity. These particles react with molecules in living organism, which are responsible for toxic process. This reaction is probably base of this mechanism [9]. Oxidative stress is undesirable process, because this is one of the main factors responsible for damage of cell membrane, for example: the erythrocyte membrane. This mechanism is based on the free radical chain reactions [10]. The production of free radicals is the main effect of this process. The free radicals are responsible for the damage of cell membrane structure and change properties of cell membrane fluidity and conformations of phospholipid bilayer. Damage of nucleus is caused by lipid peroxidation, nucleus contains a genetic materiał (DNA) of cell. Oxidative stress is the cause of the destruction of erythrocyte membrane, and this mechanism is considered as the base of developing the phenomenon of haemolysis. In the scientific world this process is called as "anemia haemolytica"-the decrease in the amount of erythrocytes in the peripheral blood from egzogenic or endogenic factors.

It has been proved by K. Mitura [8], that Diamond Powder Particles has a very high biological activity in living organism at a molecular level. The mechanism of inhibition of oxidative stress by Diamond Powder Particles in vitro was established, the newest results show the influence on free radical damage reaction in red blood cells.

\section{EXPERIMENTAL}

\section{Microwave/Radio Frequency Plasma Assisted Chemical Vapour Deposition method.}

Carbon coatings have been produced at the Institute of Materials Science and Engineering, Technical University of Lodz (Poland), by MW/RF PA CVD method [11]. The apparatus for carbon film synthesis by this method is presented in Fig. 1. In this system plasma is initiated by two source of energy working at microwave frequency and 
radio frequency. In this method it is possible to apply one or both frequencies simultaneously, or to excite of plasma by use MW or RF separately.

Microwave energy is transferred from the generator to the chamber by circulator, tuner and quartz tubing. The RF energy is supplied to the system through an isolated electrode. The system is additionally equipped with vacuum pumping, gas dosing and control modules. For the synthesis of carbon films pure methane has been used. Pure Methane, as a source of carbon, was supplied through a shower device in the vicinity of the sample.

\section{Parameters of Synthesis of Carbon Coatings}

During the film deposition flow rate of gas and RF power remained at $30 \mathrm{~cm}^{3} / \mathrm{min}$ and $300 \mathrm{~W}$, respectively. The substrates were placed on the substrate holder inside the deposition chamber by pumping down to several $\mathrm{Pa}$. The MW power used in the studies comprises a 100-1000 W range, the negative self-bias voltage was approximately $-100 \mathrm{~V}$. Depositions were carried out for various lengths of time: 10 and 30 min. Carbon coatings have been plotted on the nanofiber textile produced from the poly (vinylalcohol) and (PVA) and polyurethane (PUR), formed onto basic fiberous material - polypropylene (PP). A schema of the substrate is shown in Fig. 2.

\section{The Nanospider Technology}

Nanofiber textile has been used as the substrate produced by the modern Nanospider method. This technology was elaborated at The Technical University of Liberec (Czech Republic), within The Department of Nonwovens, and has already been patented. The technology is being further developed by Elmarco s.r.o. (also in Liberec). Elmarco has received the exclusive license for the production and sale of Nanospider on an industrial scale. This commercial method for production of polymeric nanofibres was documented by Jirsak [12].

Nanospider is a modified electrospinning method. This is a simple and versatile method for production ultra-thin fibers from a variety of materials that include polymers. This technique requires the use of a high voltage electrostatic field to create an electrically charged stream of polymer solution or melt. In a typical process, high voltage is used between a grounded collector and a capillary tube. A droplet of a liquid polymer is brought to the tip of a capillary and upon voltage application the droplet forms a Taylorcone. When the applied electric field overcomes the surface tension of the droplet, a charged jet of liquid is ejected from the tip of cone. During the jet's travel, the solvent gradually evaporates, and a charged polymer fiber is left to accumulate on the grounded target.

The innovatory idea of the Nanospider is based on the possibility of producing nanofibers from a thin layer of liquid polymer. In this case Taylor cones (the source of nanofibers) are created on the surface of a rotating roller, immersed in a polymer solution - see Fig. 3.

Because the Taylor streams are formed next to each other, throughout the entire length of the roller, this revolutionary idea produced many advantages, such how high productive ability. Nanospider has the ability to process a wide range of polymers in diameters of $50-300 \mathrm{~nm}$ into nonwoven webs of 0.1-5 gsm. 


\section{The Contact Angle Studies}

The measurements of the contact angle of carbon films were carried out in atmospheric conditions at room temperature. A droplet of distilled water with the volume of $3 \mu \mathrm{l}$ was released onto the sample surface. For each sample, at least three drops were released. Contact angle was designated by apparatus created in our institute.

\section{Structure of the Nanocomposite}

The nanocomposite structure was analyzed by scanning electron microscope (SEM). Investigation was made at the Faculty of Textile Engineering, Technical University of Liberec (Czech Republic).

\section{RESULTS}

\section{The Contact Angle Studies}

Results of contact angle investigation indicated the radical improvement of hydrophobic properties for nanofiber textiles covered by carbon coatings. Only the carbon coating/PVC (samples devoid of basic fiberous material) system has a hydrophilic property, with water droplets spread out across the surface of the polymer samples uncovered by a carbon layer; results are shown in Table 1 .

\section{Structure of the Nanocomposite}

The most essential result of this investigation is the fact that after carbon layer deposition the structure of material has not changed. The spaces between the nanofibers in the textile have not been closed by the material of the film. So, the functional properties of the nanofiber textile, mentioned below, remain unchanged.

A SEM microscopic image of carbon film deposited onto a nanofiber textile is presented in Fig. 4.

\section{APPLICATION OF NANOFIBERS}

Nanofiber material produced by Nanospider method is characterized by very good sound absorption of low frequencies, but the ability to absorb higher sound frequencies is also good. These properties make possible the use of nanofibers in automotive components, aviation, building and the machinery industries. There are excellent prospects to use this material in the following applications: acoustic and anti-noise solutions for sound studios, concert halls, lecture halls, theatres, cinemas, classrooms, stadiums, industry halls.

Some groups of nanofibers materials are characterized by fine surface and high air permeability. These properties are desirabled in hygienic products such as diapers, napkins, wipers etc. Works on developments in this area are continuing.

Nanofibers can be used also as basic material in new products with a cleaning or healing effect such as napkins, wipes or linen. The structure of this material is perfect to allow the placing between the nanofibers of solid or liquid particles. It is predicted that 
these nanocomposites will be more biocompatible with the human body, particularly in contact with sensitive areas like the nose and the eyes.

Nanofiber textiles are predicted for use as material for wound dressing. Their use makes possible the transport of oxygen and exudates, and at the same time protects from bacteria. Moreover, Nanofiber textiles can be used as carrier for some antimicrobial, haemostatic or healing enhancement pharmaceuticals. There is great hope that in the future medicine will be able to use Nanofibers in the reconstruction of skin, bones, muscles, veins and nerves. Because the distance between fibers is smaller than the dimensions of bacteria, the virus of influenza, and Human Immunodeficiency Virus (HIV) for example, use of this material as separation membranes with defined properties, intelligent filters with antibacterial effects or antidotes is considered possible.

\section{CONCLUSIONS}

In summary, we have shown that the carbon coatings can be deposited on the nanofiber textile produce by the Nanospider method at room temperature using the MW/RF PACVD technique.

The hydrophobic character of the covered surfaces has been proved. This fact confirms content of diamond phase in produced carbon coatings. This modification of nanofiber textile has not influenced the characteristic properties of substrate, the spaces between the nanofibers have not been closed by material of the film. Distance between fibers is very small, smaller than the dimensions of bacteria, the virus of influenza, or Human Immunodeficiency Virus (HIV) for example. Therefore this material is used as a filter in many cases, especially in medical applications.

Moreover, it has to be underline that before synthesis of carbon coatings, nanofiber textile was working as mechanical filter. Deposition of carbon coatings makes possible the activation of nanofiber textile, in this way we produced active filter.

More investigations are needed to check the influence of carbon coatings on the properties of nanofiber textile produced by the Nanospider method, especially from point of view of medicine.

\section{ACKNOWLEDGEMENTS}

This study was financed by MSM4674788501.

\section{REFERENCES}

[1] Fernandes, A.J.S., Salgueiredo, E., Oliveira, F.J., Silva, R.F., Costa, F.M., "Directly MPCVD diamond-coated $\mathrm{Si}_{3} \mathrm{~N}_{4}$ disks for dental applications.", Diamond and Related Mat. No.14, pp. 626-630, (2005)

[2] Rodil, S.E., Olivares, R., Arzate, H., Muhl, S., "Properties of carbon films and their biocompatibility using in-vitro tests.", Diamond and Related Mat., No.12, pp. 931937, (2003).

[3] Okrój, W., Kamińska, M., Klimek, L., Szymański, W., Walkowiak, B., "Blood platelets in contact with nanocrystalline diamond surfaces.", Diamond and Related 
Mat., No. 15, pp. 1535-1539, (2006).

[4] Dorner-Reisel, A., Schürer, Ch., Müller, E., "The wear resistance of diamond-like carbon coated and uncoated Co28Cr6Mo knee prostheses.", Diamond and Related Mat., No. 13, pp. 823-827, (2004).

[5] Mitura, S., Niedzielski, P., Jachowicz, D., Langer, M., Marciniak, J., Stanishevsky, A., Tochitsky, E., Louda, P., Couvrat, P., Denis, M., Lourdin, P., "Influence of carbon coatings origin on the properties important for biomedical application.", Diamond and Related Mat., No. 5, pp. 1185-1188, (1996).

[6] Yasuharu, O., Hirakuri, K., Tsuchimoto, K., Friedbacher, G., Miyashita, O., "Uniform deposition of diamond-like carbon films on polymeric materials for biomedical applications.", Surf. and Coatings Tech., No. 184, pp. 263-269, (2004)

[7] Elinson, V.M., Sleptsov, V.V., Laymin, A.N., Potraysay, A.N., Kostuychenko, L.N., Moussina, A.D., "Barrier properties of carbon films deposited on polymer-based devices in aggressive environments.", Diamond and Related Mat., No. 8, pp. 2103-2109, (1999).

[8] Bakowicz-Mitura, K., Bartosz, G., Mitura, S., "Influence of diamond powder particles on human gene expression.", Surf. and Coatings Tech., No. 201, pp. 6131-6135, (2007).

[9] Bakkowicz, K., Mitura, S., "Biocompatibility of NCD.", J. of Wide Bandgap Mat., No. 9, pp. 261-272, (2002).

[10] Mitura, K., Niedzielski, P., Bartosz, G., Moll, J., Walkowiak, B., Pawłowska, Z., Louda, P., Kieć-Świerczyńska, M., Mitura, S., "Interactions between carbon coatings and tissue.", Surf. and Coatings Tech., No. 201, pp. 2117-2123, (2006).

[11] Kaczorowski, W., "Synthesis of ultrananocrystalline diamond by use dual freuqency plasma.", in edited by Mitura, S., Niedzielski, P., Walkowiak, B., "New technology for medical applications: studying and production of carbon surfaces allowing for controllable bioactivity.", NANODIAM, PWN (2006) pp. 41-45.

[12] Jirsak, O., Sanetrnik, F., Lukas, D., Kotek, V., Martinova, L., Chaloupek, J., WO2005-024101 (2005), Czech. Pat. 
Table 1. Results of contact angle investigation

\begin{tabular}{|c|c|c|c|c|}
\hline $\begin{array}{c}\text { kind of } \\
\text { substrate }\end{array}$ & $\begin{array}{c}\text { PUR with basic } \\
\text { fiberous } \\
\text { material }\end{array}$ & $\begin{array}{c}\text { PUR witout } \\
\text { basic fiberous } \\
\text { material }\end{array}$ & $\begin{array}{c}\text { PVA with basic } \\
\text { fiberous } \\
\text { material }\end{array}$ & $\begin{array}{c}\text { PVA without } \\
\text { basic fiberous } \\
\text { material }\end{array}$ \\
\hline character & hydrophobic & hydrophobic & hydrophobic & hydrophilic \\
\hline
\end{tabular}

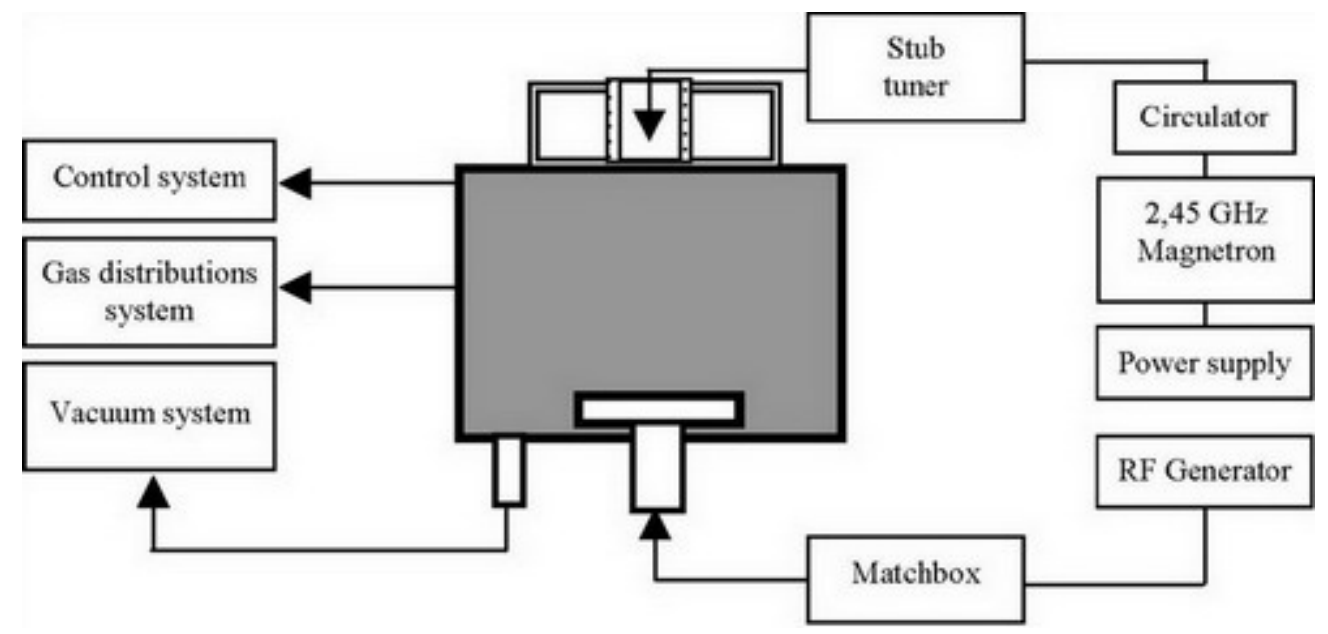

Fig. 1. Schematic of the apparatus for carbon film synthesis by MW/RF PACVD decomposition [11].

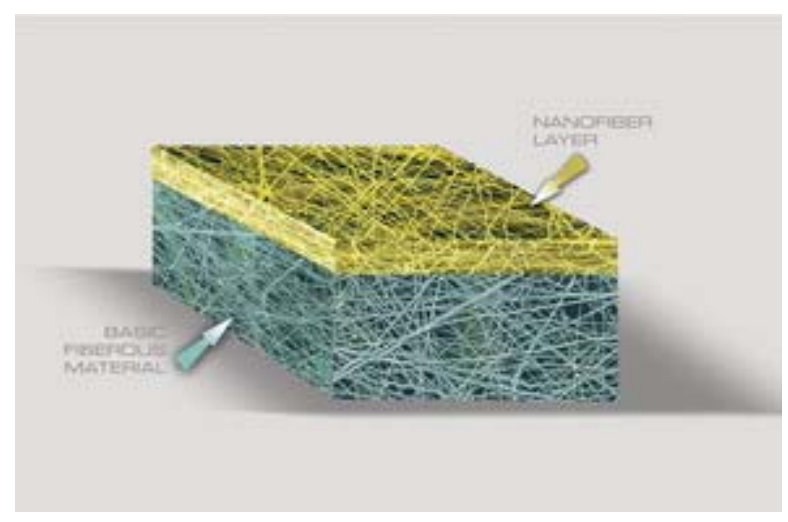

Fig. 2. Nanofiber textile onto basic fiberous material. 


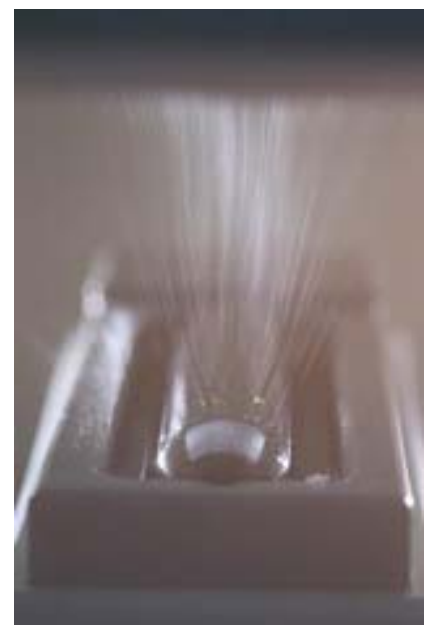

Fig. 3. Rotating roller, immersed in a polymer solution

a)

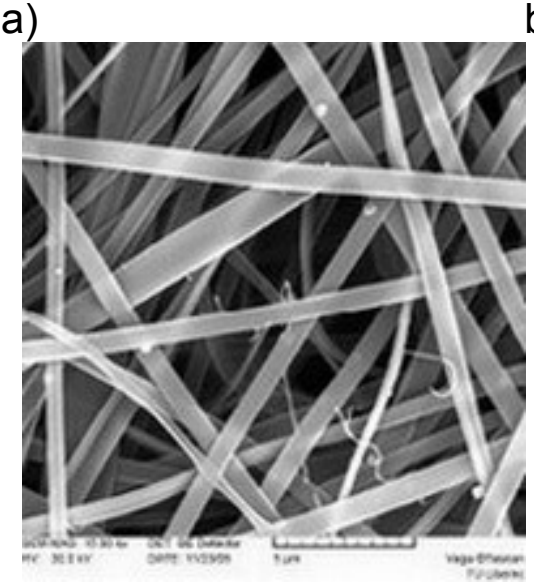

b)

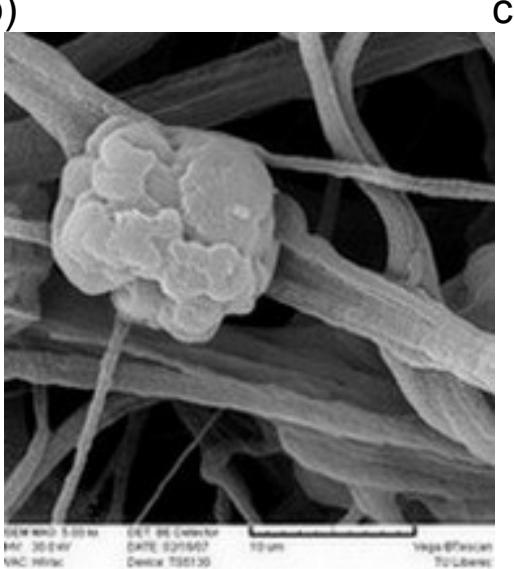

c)

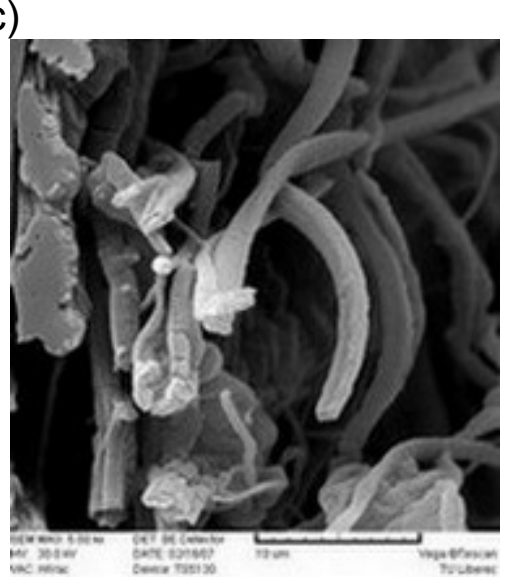

Fig. 4. SEM image of nanofiber textile a) uncovered, b) covered by carbon coatings, c) cross section of nanofibers covered by carbon coatings 